\title{
Transient Response of 50 KiloAmp Y-Ba-Cu-O Rings and Ring Pairs to Pulsed Magnetic Fields
}

\author{
Thomas R. Askew and Yung S. Cha
}

\begin{abstract}
Shielding current limits and magnetic diffusion characteristics have been measured at $77 \mathrm{~K}$ in large-grain YBCO rings with oriented microstructures. The samples are surrounded by a drive coil that can achieve magnetic fields in excess of 1 Tesla and induce currents in excess of $50 \mathrm{KAmps}$ when driven by current pulse of a few msec duration. Simultaneous magnetic measurements with a Rogowski Coil and a Hall Probe allow determination of the induced current in the sample and the field in the center of the sample. These measurements show that field penetration occurs in a complex way that includes delays and transient effects caused by magnetic diffusion and sample heating. Dramatic threshold effects are observed that are probably related to a creep-flow transition coupled with local heating effects. Geometric effects are investigated using a single drive coil and a pair of YBCO rings with various spacings. A test geometry is used that is equivalent to a simple penetration-type inductive fault current limiter, and the experimental results are therefore of interest for design and characterization of these devices.
\end{abstract}

Index Terms- Fault-current limiter, magnetic diffusion, magnetic shielding, pulsed current testing.

\section{INTRODUCTION}

$\mathrm{R}_{\mathrm{tan}}^{\mathrm{t}}$ ECENT advances in $\mathrm{YBa}_{2} \mathrm{Cu}_{3} \mathrm{O}_{7}$ melt process technology have enabled the fabrication of parts that have oriented microstructures on the length scale of $2-10 \mathrm{~cm}$ and conduction properties in the $a-b$ plane that are essentially free of the weak-link coupling behavior seen in sintered YBCO [1]-[5]. When parts of this type are subjected to pulsed magnetic fields they exhibit transient effects [6] and magnetic diffusion [7],[8] similar to behavior previously observed in $\mathrm{Bi}_{2} \mathrm{Sr}_{2} \mathrm{CaCu}_{2} \mathrm{O}_{\mathrm{X}}$ tubes. These previous observations can be described in terms of classical magnetic diffusion (magneto-quasistatic form of Maxwell's equations, with an Ohmic conductor) with judicious use of Bean's critical state model [8]. The introduction of a more realistic non-Ohmic E-J curve into the theory leads to a nonlinear diffusion equation [7].

The reported measurements are used to compare the diffusive and dissipative behavior of $\mathrm{YBCO}$ and $\mathrm{BSCCO}$ parts under similar conditions at $77 \mathrm{~K}$ and to evaluate the possible application of these particular YBCO rings in fault current limiters (FCLs) for the electric power industry [9],[10]. The essential parts of an inductive FCL are an iron

Manuscript received September 17, 2000. This work was supported in part by the U.S. Department of Energy (DOE), Energy Efficiency and Renewable Energy, as part of a DOE program to develop electric power technology, under Contract W-31-109-Eng-38.

Y. S. Cha is with Energy Technology Div., Argonne National Laboratory, Argonne, IL 60439 (telephone: 630-252-5899, e-mail: yscha@anl.gov).

T. R. Askew is with Kalamazoo College, Kalamazoo, MI 49006 (telephone: 616-337-7097, e-mail: askew@kzoo.edu). core or yoke located inside a superconductor shielding tube and a copper drive coil wound on the outside of the superconductor tube. When an FCL is used under normal operating conditions the impedance is low because the magnetic flux from the drive coil is shielded by the superconductor and only weakly coupled into the iron core. Under fault conditions, the large current in the copper drive coil produces a magnetic field that exceeds the shielding capability of the superconductor tube. This large field then penetrates into the iron core, couples into the yoke, and causes the impedance of the drive coil to rise sharply. Under voltage-limited conditions, the increase in impedance causes the current in the drive coil to drop, thereby achieving a useful current-limiting effect that can be applied in power distribution systems.

\section{EXPERIMENTAL DETAILS}

\section{A. YBCO Parts}

The rings used in this study were made by a top-seeded melt-growth process that is described in detail elsewhere [2],[3]. The large dimension of the rings (in comparison to previous work) required the use of multiple seeds to achieve orientation of the multiple growth domains. The rings are in the form of a torroid with a $2 \mathrm{~cm} \times 2 \mathrm{~cm}$ square crosssection. The rings have an outside diameter of $10 \mathrm{~cm}$, an inside diameter of $6 \mathrm{~cm}$, and a height of $2 \mathrm{~cm}$. The size of the growth domains is on the same order as the crosssection, which leads to a complex morphology that varies with position around the ring. Measurements that are reported later reveal a critical current density of about 10 $\mathrm{KAmps} / \mathrm{cm}^{2}$ and a critical current around the ring of about $40 \mathrm{KAmps}$. These are well above the properties expected for high quality, unoriented, sintered $\mathrm{YBCO}$ at $77 \mathrm{~K}$, and indicate that the grain alignment within each domain must be quite good, and the losses at the domain edges no worse than grain-to-grain coupling in the best sintered material.

\section{B. Test Geometry and Measurement Methods}

The test configuration is motivated by the geometry of an inductive FCL, and is shown in cross-section in the scale drawing below: 


\section{DISCLAIMER}

This report was prepared as an account of work sponsored by an agency of the United States Government. Neither the United States Government nor any agency thereof, nor any of their employees, make any warranty, express or implied, or assumes any legal liability or responsibility for the accuracy, completeness, or usefulness of any information, apparatus, product, or process disclosed, or represents that its use would not infringe privately owned rights. Reference herein to any specific commercial product, process, or service by trade name, trademark, manufacturer, or otherwise does not necessarily constitute or imply its endorsement, recommendation, or favoring by the United States Government or any agency thereof. The views and opinions of authors expressed herein do not necessarily state or reflect those of the United States Government or any agency thereof. 


\section{DISCLAIMER}

Portions of this document may be illegible in electronic image products. Images are produced from the best available original document. 


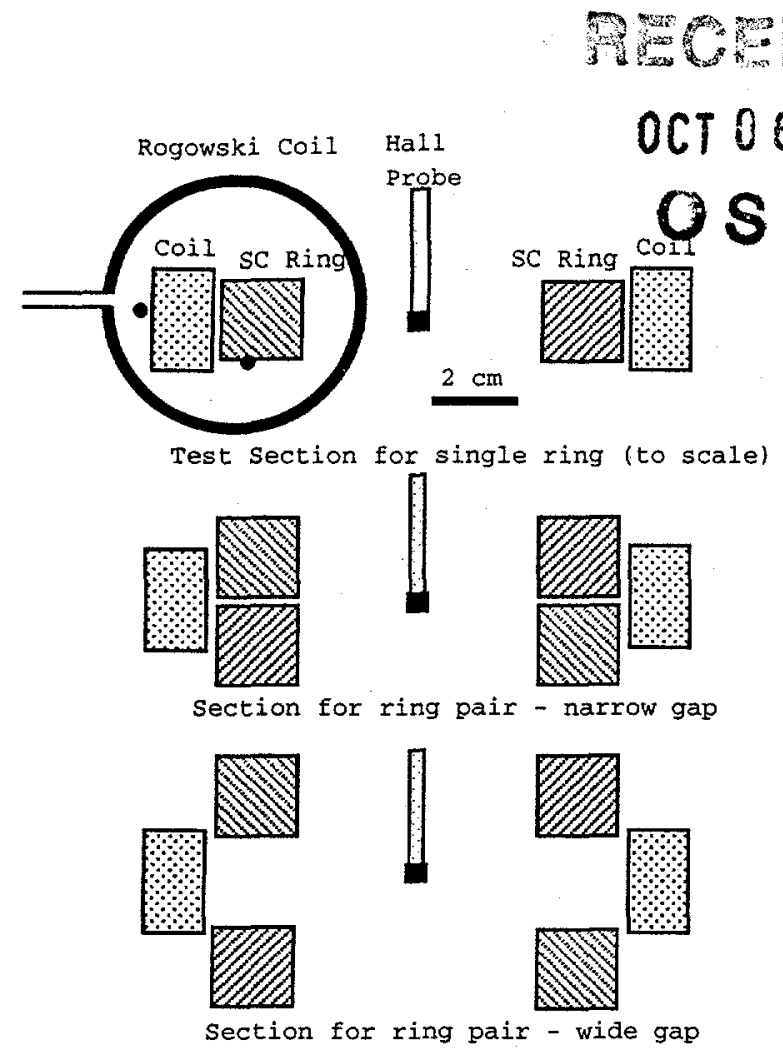

Fig. 1. Cross-section of test configurations, drawn to scale.

The drive coil consists of 200 turns of AWG 16 copper wire, has a rectangular cross-section, $2.5 \mathrm{~cm}$ high, and 1.5 $\mathrm{cm}$ in the radial direction, and produces a magnetic field of $1.94 \mathrm{mTesla} / \mathrm{amp}$ at its center. The coil inner dia. is 10.4 $\mathrm{cm}$, which leads to a $0.2 \mathrm{~cm}$ gap between the I.D. of the coil and the O.D. of the rings. The coil is driven by a capacitivedischarge pulsed current supply that is described in detail elsewhere [8],[13]. Typical charging voltages for the capacitor bank would be $50-150$ Volts on 0.24 Farads. Depending on the conditions, this might produce current pulses in the 60-600 Amp range, producing magnetic field strengths up to 1.1 Tesla for a few tens of milliseconds.

The axial magnetic flux density at the center of the apparatus is measured by a high-bandwidth Hall Probe. A shunt connected in series with the drive coil allows accurate measurement of the drive current. The integrated signal from a Rogowski Coil is used in the conventional way $[11,12]$ to calculate the sum of the current flowing in the drive coil and superconducting ring(s). Since the drive coil current is measured separately, the current induced in the ring(s) can be obtained by subtraction. All measurements (Hall voltage, shunt voltage, Rogowski coil voltage) are made with 16 bit A/D conversion at time constants that are much shorter than any phenomena in the pulse characteristics or test system response.

\section{RESULTS AND DISCUSSION}

\section{A. Single Ring Tests}

Figs. $2 \mathrm{a}$ and $2 \mathrm{~b}$ show tests of a single ring, with drive current in the coil (in KAmp-turns) and the induced current in the ring (KAmps) indicated on the left axis. The measured magnetic flux density $\mathrm{B}$ at the center of the structure is indicated on the right axis.

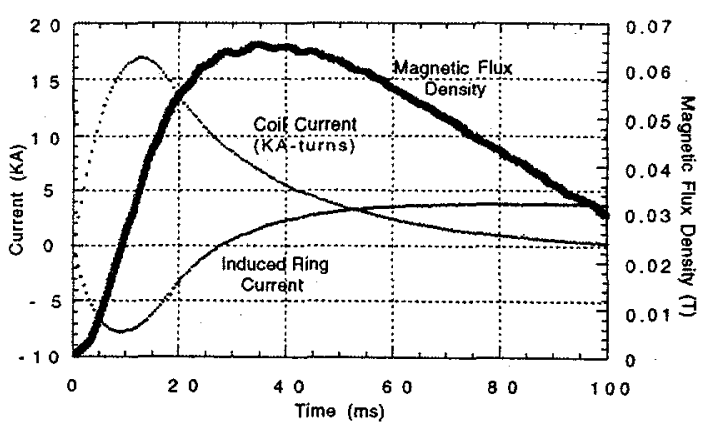

Fig.2a. Coil current, induced ring current, and axial magnetic flux density for a single YBCO ring.

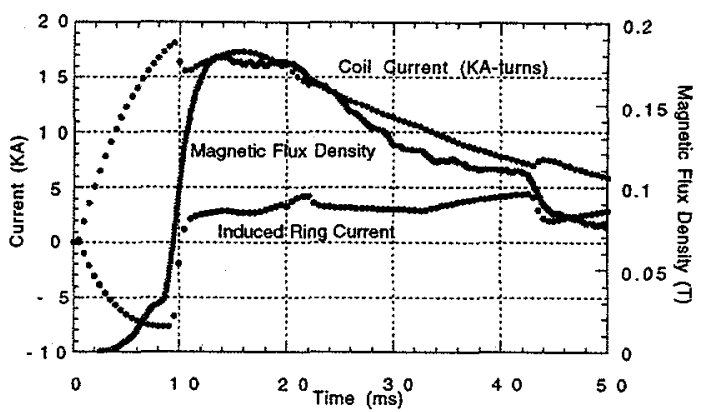

Fig.2b. Similar data for the same ring as above, with a slightly larger current pulse.

The response in fig. $2 \mathrm{a}$ is similar to that previously observed in $\mathrm{Bi}_{2} \mathrm{Sr}_{2} \mathrm{CaCu}_{2} \mathrm{O}_{\mathrm{x}}$ and described in detail elsewhere [8]. During the first few milliseconds of the current pulse, the magnetic field produced by the drive coil roughly cancels the field produced by the induced current in the ring, resulting in a very small measured field at the center of both rings. Previous studies have focused on hollow cylindrical structures (tubes) which produce magnetic filed distributions similar to a solenoid of equivalent dimensions. In the center of such structures, the axial flux density is weakly dependent on the cylinder radius (short coil formula) or in the limit of the infinite coil approximation, independent of the cylinder radius. Thus, a drive coil and a coaxial cylindrical superconducting cylinder can produce canceling fields at the center while each carries the same current (measured in ampturns) but on a different radius. These equal and opposing currents are sometimes called mirror-image currents, and are observed in practice [13]. In this case the $\mathrm{YBCO}$ rings act more like a single round turn of wire, and produce an axial flux density at the center that is roughly proportional to the inverse of the radius of the loop. Since the ring has a much smaller mean radius, it would be expected to carry a mirror current related to the larger drive coil current by the ratio $3: 2$. The observed ratio is more like 2:1, with some of the difference coming from flux leakage through the gap $(0.2 \mathrm{~cm})$ between the drive coil and the ring.

When the drive coil current is peaking at about $12 \mathrm{msec}$, the magnetic field is still largely excluded from the interior of the ring, resulting in a measured flux density of $35 \mathrm{mT}$ at that time. The value of the applied magnetic field $\mu_{0} \mathrm{H}$ at that time is $170 \mathrm{mT}$, showing that penetration has not occurred. The measured flux density at the center reaches a maximum about $23 \mathrm{msec}$ after the two circulating currents have peaked, showing the same signature of magnetic 
diffusion seen in BSCCO structures earlier [8]. The crossover of the induced current to positive values (that is beginning to flow in the same direction as the original drive current) and the resulting measured flux density can also be explained by these studies. As in earlier studies, the measured magnetic flux density at the center follows what would be predicted from the difference of the two currents, although in this case some adjustment for differing radii is necessary. The ripple near the peak in the measured field is $60 \mathrm{~Hz}$ power line interference, and not associated with the sample.

Fig. $2 b$ illustrates the effect of an increase in the coil current from a maximum of $17 \mathrm{KAmp}$-turns (fig. 2a) to a maximum of $18 \mathrm{Kamp}$-turns. At $9 \mathrm{msec}$ a dramatic transition occurs in which most of the current flowing in the ring dissipates, causing a sudden boiling of the liquid $\mathrm{N}_{2}$ bath and a significant back e.m.f. which opposes the drive voltage of the capacitor bank, quickly reducing the current in the drive coil. During the next three msec a large magnetic flux density penetrates to the center of the structure, resulting in a measured $B$ of $0.18 \mathrm{~T}$ which is the same as the value of $\mu_{0} \mathrm{H}$ calculated from the coil constant and the peak value of the drive current. The transition at $42 \mathrm{msec}$ is associated with a safety feature of the pulsing system that protects the electrolytic capacitor bank from being charged up backwards. The conditions used for fig $2 a$ and $2 b$ were repeated alternately many times on the same ring, with no observed change in the data or apparent damage to the sample.

Magnetic field penetration in several $\mathrm{msec}$ and similar sample heating have been observed in a number of different BSCCO cylinders [8],[13], but never with this abruptness. The ring used for the figures above contained a radial fissure caused by thermal contraction during cooling, and the resulting defect intersected about $1 / 3$ of the cross-sectional area. Since the critical current was clearly lower at that spot than elsewhere on the ring circumference, the abruptness of the transition is likely caused by a flux creep-to-flux flow transition at that spot, rapidly producing enough heat to drive the spot normal.

An additional pair of YBCO rings were obtained, similar in dimension to the one discussed above, but without the defect. The two rings behaved similarly, and the response for one of them is shown in fig. 3.

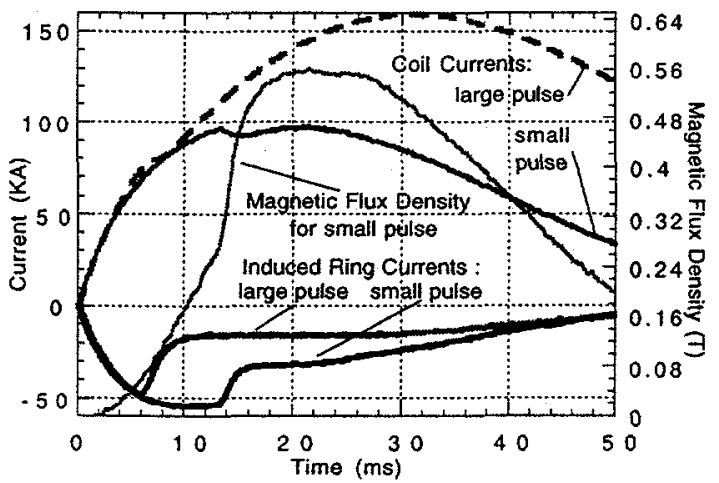

Fig. 3. Currents induced in a YBCO ring when driven just to the point of dissipation by a coil current pulse, marked "small pulse". Driving the system harder produces a second set of curves marked "large pulse".
The first set of curves, marked "small pulse" are produced when the system is driven just to the point of dissipation, leading to an induced current of $54 \mathrm{KA}$. Curves are shown for the current pulse in the drive coil, the induced current in the YBCO ring, and the measured magnetic flux density at the center. The crossover from magnetic diffusion with little thermal dissipation to field penetration with dissipation can be seen at $14 \mathrm{msec}$, where the slope of the magnetic flux density curve changes.

If the system is driven with a coil current pulse slightly smaller than this, it reverts to the type of behavior illustrated in fig. $2 \mathrm{a}$, but at currents that are 5 times larger than those shown in that figure. If the system is driven much harder, using the coil current pulse marked "large", the result is the transition at $6 \mathrm{msec}$. Violent boiling of liquid $\mathrm{N}_{2}$ occurs under this condition, but the response of the system is far less abrupt than that of fig. $2 b$, despite the much larger currents. This supports the conclusion that these latter two rings do not contain significant weak spots which reduce the critical current at particular points in the circumference. The result is a more uniform energy dissipation pattern coupled into a lot more ring mass.

Coil current pulses which fall between the "large" and "small" curves produce a family of induced current pulses falling between the two curves shown.

The data of fig. 3 indicate that the ring has a critical current of about $50 \mathrm{KA}$, and a critical current density of about 12 $\mathrm{KA} / \mathrm{cm}^{2}$. Since these have been measured under dynamic conditions, it is not clear how these values relate to the static "equilibrium" critical current measured with the $1 \mathrm{microvolt} / \mathrm{cm}$ criterion. This question is explored elsewhere and data are available for BSCCO that show little difference between the various methods of measurement because the differential resistivity rises rapidly at the transition [8].

\section{B. Ring Pair Tests}

These rings can support critical currents (at least under dynamic conditions) that are large enough to be useful for application in electric power distribution networks. In actual application the rings are likely to be used in coaxial arrays, prompting us to test a pair of rings with a variable gap in between. The cross-sections are shown in fig. 1, with a "narrow gap" of $0.2 \mathrm{~cm}$, and a "wide gap" of $2.0 \mathrm{~cm}$. Figure 4 below shows the behavior of the ring pairs with variation in the gap.

Behavior similar to that observed in fig. $2 a$ is shown, but at much higher drive currents. The magnetic field is able to intrude between the coils, and reach the Hall probe in the center, but with the very long time constants (in this case about 50 and $60 \mathrm{msec}$ ) associated with magnetic diffusion. The variation in time constant is not surprising because the rings have a rough $\mathrm{c}$ axis orientation parallel to the main axis of the ring. This means that in the single ring case (fig. 2a) the field front propagates into the sample in a direction perpendicular to the $c$ axis, thus inducing ring currents primarily in the $a-b$ plane. Particularly for the wide gap case, the field front propagates in on an angle to the $c$ axis (roughly parallel to the hash lines in fig. 1) thus inducing currents which must propagate at least partially in the c direction. 


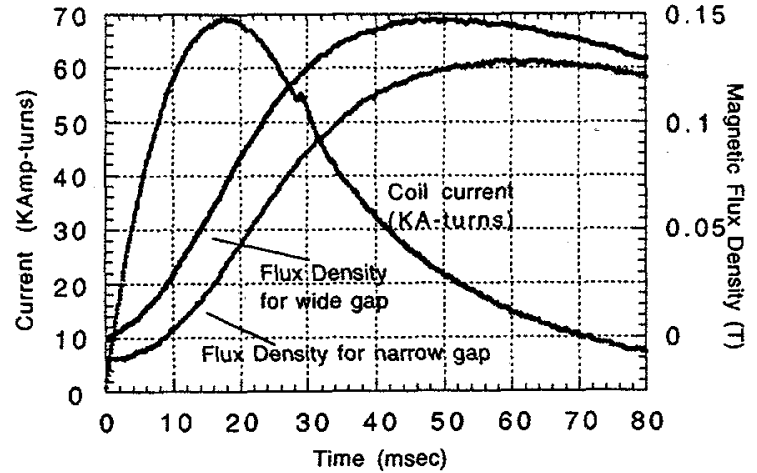

Fig. 4. Coil current and axial magnetic flux density for ring pairs with a variable gap.

Geometric and demagnetization effects are, no doubt, important as well. It is not surprising that the wider gap allows a higher field at the center. The ring pair is shielding the vast majority of the applied magnetic field in either case, as $\mu_{0} \mathrm{H}$ at the peak current is $0.68 \mathrm{~T}$. The data shown are not virgin pulses, but rather follow after earlier pulses of similar size. This distinction matters because the rings may retain a small trapped magnetic field after the pulse. In this case the narrow gap geometry retains a small trapped field in the opposite direction to the original applied field, and this appears as a small negative initial value for its plot in fig. 4 . The larger gap does not show this field retention, probably due to the differing demagnetization factors for the two cases. In any event, retained field does not appear to be a significant problem for applications.

\section{CONCLUSIONS}

YBCO rings have been tested individually and in pairs under conditions likely to be encountered in application-scale inductive fault current limiters. The rings were found to support critical currents of about 50 KAmps under dynamic conditions similar to those in a 50 or $60 \mathrm{~Hz}$ power distribution grid.
Magnetic diffusion phenomena similar to those previously seen in BSCCO systems were observed. Rapid transitions from flux creep to flux flow were observed, and these transitions were observed to change when the sample contained an obvious weak link. The rings functioned well in pairs, and the samples survived a number of thermally violent transitions into dissipative electrical conduction. Future work will likely involve similar experiments using 60 $\mathrm{Hz} \mathrm{AC}$ current.

\section{ACKNOWLEDGMENT}

The authors thank J. A. Lockwood of Ford Motor Company for his help in developing the data acquisition system. J. R. Gaines and S. Sengupta of Superconductive Components, Inc. are thanked for useful discussions and fabrication of the YBCO rings.

\section{REFERENCES}

[1] I. Monot, J. Wang, M.P. Delamare, J. Provost, and G. Desgardin, Physica C, vol. 267, p. 173, 1996.

[2] S. Sengupta, D. Shi, J.S. Luo, A. Buzdin, V. Gorin, V.R. Todt, C. Varanasi, and P.J. McGinn, J. Appl. Phys., vol. 81, p. 7396, 1997.

[3] S. Sengupta, J. Corpus, J.R. Gaines, V.R. Todt, X.F. Zhang, D.J. Miller, C. Varanasi, and P.J. McGinn, IEEE Trans. Appl. Supercond., vol. 7, no. 2, p. 1723, 1997.

[4] A. Khalfi, G. Trolliard, B. Soulestin, D.S. Smith, J.P. Bonnet, D. Bourgault, and R. Tournier, J. Mater. Res., vol. 13, p. 2800, 1998.

[5] C.J. Kim, and G.W. Hong, Supercond. Sci. Technol., vol. 12, p. R27, 1999.

[6] U. Mizutani, T. Oka, Y. Itoh, Y. Yanagi, M. Yoshikawa, and H. Ikuta Appl. Stpercond., vol. 6, p. 235, 1998.

[7] Y.S. Cha, Physica C, vol. 330, p.1,2000

[8] Y.S. Cha and T.R. Askew, Physica C, vol. 302, p.57, 1998.

[9] V. Meerovich, V. Sokolovsky, G. Jung, and S. Goren, IEEE Trans. Appl. Supercond., vol. 5, no. 2, p. 1044, 1995. (and other papers from the same session)

[10] J. R. Cave, D. Willen, R. Nadi, W. Zhu, A. Paquette, R. Boivin, and Y. Brissette, IEEE Trans. Appl. Supercond., vol. 7, no. 2, p.832, 1997.

[11] J. Ramboz, IEEE Trans. Instr, and Meas., vol. 45, no. 2, p.511, 1996.

[12] L. Kojovic, IEEE Computer Appl. in Power, vol. 10, no. 3, p.47, 1997.

[13] Y.S. Cha, D.J. Evans, and J.R. Hull, IEEE Trans. Appl. Supercond, vol. 9, no. 2, p. 1320, 1999. 